\title{
Multi-epoch Observations of LMXBs in Early-type Galaxies
}

\author{
Gregory R. Sivakoff ${ }^{1}$, Andrés Jordán ${ }^{2,3}$, Adrienne M. Juett ${ }^{1}$, \\ Craig L. Sarazin ${ }^{1}$, and Jimmy A. Irwin ${ }^{4}$ \\ ${ }^{1}$ Department of Astronomy, University of Virginia, P. O. Box 3818, \\ Charlottesville, VA 22903-0818, USA \\ email: grs8g@virginia.edu, ajuett@virginia.edu, sarazin@virginia.edu \\ ${ }^{2}$ European Southern Observatory, Karl-Schwarzschild-Str. 2 \\ 85748 Garching bei München, Germany; \\ email: ajordan@eso.org \\ ${ }^{3}$ Astrophysics, Denys Wilkinson Building, University of Oxford, \\ 1 Keble Road, Oxford, OX1 3RH, UK \\ ${ }^{4}$ Department of Astronomy, 909 Dennison Building, University of Michigan, \\ Ann Arbor, MI 48109-1042, USA; \\ email: jairwin@umich.edu
}

\begin{abstract}
Chandra observations of early-type galaxies have resolved large populations of lowmass X-ray binaries (LMXBs) in early-type galaxies. The majority of these observations have been snapshots on the order of a day or less. In our own Galaxy, LMXBs are known to exhibit a range of luminosity and spectral variability. Multi-epoch observations of early-type galaxies are just beginning to explore the regime of variability on timescales of days to years. We present results for NGC 4365 and NGC 4697, and compare them to the Milky Way.
\end{abstract}

Keywords. binaries: close, galaxies: elliptical and lenticular, galaxies: individual (NGC 4365), galaxies: individual (NGC 4697), X-rays: binaries, X-rays: galaxies.

\section{Introduction}

Milky Way (MW) low mass X-ray binaries (LMXBs) have been studied since the birth of X-ray astronomy (Giacconi et al. 1962). However, it is only with the sub-arcsecond resolution of the Chandra X-ray Observatory that the nature of the X-ray emission in early-type galaxies (E/S0s) can be studied in detail and that the very presence of LMXBs, suggested from spectral results by Kim, Fabbiano, \& Trinchieri (1992), could be verified. Starting with the Chandra observation of NGC 4697 (Sarazin, Irwin, \& Bregman 2000, 2001), the majority of X-ray emission in X-ray faint E/S0s has been resolved into sources whose properties are consistent with LMXBs.

Studies of LMXBs in the MW and in E/S0s are very complementary. Galactic LMXBs can be studied in great detail during both active $\left(L_{x} \gtrsim 10^{36} \mathrm{ergs} \mathrm{s}^{-1}\right)$ and quiescent $\left(\lesssim 10^{34} \mathrm{ergs} \mathrm{s}^{-1}\right)$ states across all wavelengths. From this, binary properties (e.g., donor type, compact object mass, orbital period, jet presence) can be determined, allowing for a better understanding of LMXB formation and evolution. However, there are several limitations in studying Galactic LMXBs: source distances are known for only a small subset, it is difficult to observe the whole Galaxy at once, absorption columns vary from source to source, and the size of the observed sample is limited.

For E/S0s, typical observations can only detect bright $\left(\gtrsim 10^{37} \operatorname{ergs~s}^{-1}\right)$, active LMXBs and such LMXBs cannot be studied in as great detail as in the MW. However, there 

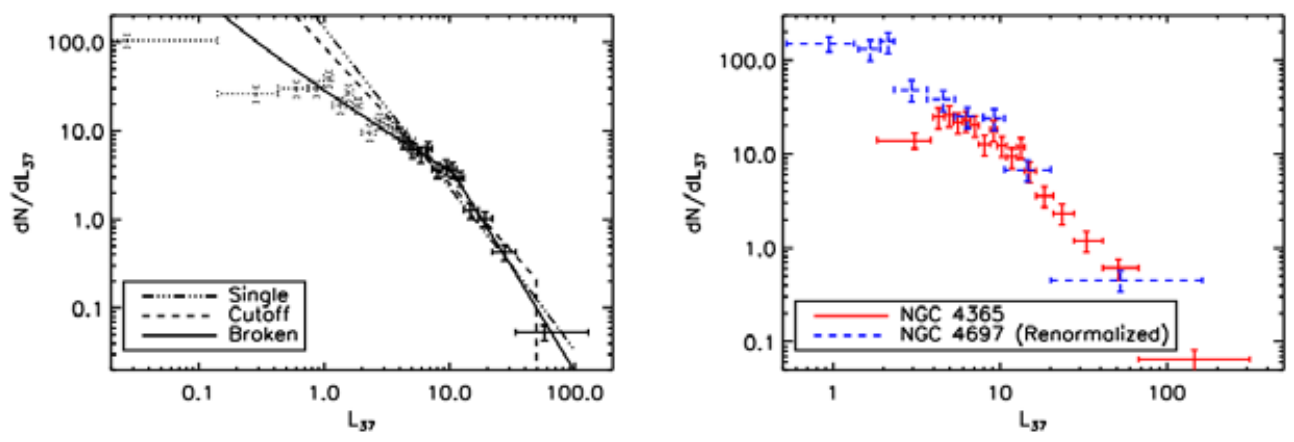

Figure 1. (Left) Completeness-corrected, instantaneous, differential luminosity function of five observations of NGC 4697. Only bins with solid lines (see text) were fit with the three power-laws displayed. (Right) A comparison of the differential luminosity function of the sum of all the observations of NGC 4365 and NGC 4697 (renormalized).

are distinct advantages to E/S0 observations. Since all LMXBs in an E/S0 share a common absorption column and distance, and most LMXBs in a nearby E/S0 fit in the field-of-view of Chandra, instantaneous luminosity functions (LFs) can be easily determined. Furthermore, E/S0s often have 50-200 sources brighter than $\sim 5 \times 10^{37} \mathrm{ergs} \mathrm{s}^{-1}$, as opposed to the few bright sources in the Galaxy.

\section{Chandra \& Hubble Observations of NGC 4697 \& NGC 4365}

With this complementary nature in mind, we have performed multi-epoch observations of NGC 4697 and NGC 4365. Four new observations per galaxy increase the total exposure time on these galaxies from $\sim 40 \mathrm{ks}$ for NGC 4697 (Sarazin, Irwin, \& Bregman 2000, 2001) and NGC 4365 (Sivakoff, Sarazin, \& Irwin 2003) to 200 ks each. These observations were designed to not only detect fainter LMXBs, but also open up the regime of LMXB variability studies in E/S0s through year timescales. The completed multi-epoch study of NGC 4697 is discussed in detail in Sivakoff et al. (2005, in preparation). The fifth and final observation of NGC 4365 is scheduled for 2005 November. Hubble observations of the centers of these galaxies (Côté et al. 2004; Jordán et al. 2004, 2005 in preparation), reveal the globular clusters (GCs), which are known to harbor large fractions of LMXBs (e.g., Angelini, Loewenstein, \& Mushotzky 2001; Sarazin et al. 2003). Flanking fields of both galaxies will be observed in HST Cycle 14.

\section{LMXB Luminosity Functions}

Standardized reduction and flare removal were performed on all observations. With the increased exposures of the combined observations, $185 \mathrm{ks}$ (NGC 4697) and $157 \mathrm{ks}$ (NGC 4365), we detected 158 (NGC 4697) \& 284 (NGC 4365) sources using CIAO WAVDETECT. For NGC 4697, we determined the count rates from PSF-scaled extraction source regions and the local background. With these photometric count rates, we determined the luminosities (assuming a $9.09 \mathrm{keV}$ bremsstrahlung spectrum and correcting for vignetting, the PSF, and QE degradation) in individual observations. Since detection was performed in the combined observation of NGC 4697, we determined completeness from the combined counts of the five observations. We created the completeness-corrected, instantaneous, differential LF (Fig. 1 Left) and fit the sum of power-law models, $d N / d L \propto L^{-\alpha}$, 
and the background LF from Kim et al. (2004), for $4.0 \times 10^{37}<L_{X}<1.3 \times 10^{39} \mathrm{ergs} \mathrm{s}^{-1}$. A single power-law in that luminosity range was rejected at $99.5 \%$, which also rejects cutoff power-laws with cutoffs beyond the upper luminosity bin. A cutoff power-law with a cutoff at $4.9 \pm 0.6 \times 10^{38} \mathrm{ergs} \mathrm{s}^{-1}$ and $\alpha=1.5 \pm 0.1$ was only marginally rejected (88\%). We note that Kim \& Fabbiano (2004) found evidence for a possible break in the LF at this luminosity. A broken power-law was an acceptable fit with a break at $1.1 \pm 0.2 \times 10^{38} \mathrm{ergs} \mathrm{s}^{-1}, \alpha_{l}=0.8 \pm 0.3$, and $\alpha_{h}=2.4 \pm 0.2$. We note that the broken power-law also goes through many of the low luminosity data points that we did not fit.

Since the observations of NGC 4365 are unfinished, we only roughly compare its LF to that of NGC 4697. In Fig. 1 (Right), we display the differential LF of the combined observations without completeness corrections, renormalizing the NGC 4697 LF by matching the number of sources with $L_{X}>10^{38} \mathrm{ergs} \mathrm{s}^{-1}$ with that of NGC 4365. The rough shapes of the LFs match. Since NGC 4365 has almost twice as many sources as NGC 4697, we should be able to probe the LF of NGC 4365 in greater detail than that of NGC 4697.

\section{GC-LMXB Connection}

One emerging pattern of the GC-LMXB connection in E/S0s is that above $10^{38} \mathrm{ergs} \mathrm{s}^{-1}$, $\approx 4 \%$ of GCs contain an LMXB (Sarazin et al. 2003). With deeper observations we can explore this connection at lower luminosities. For instance, in NGC 4697, at our $3 \sigma$ detection limit of $\approx 1.4 \times 10^{37} \mathrm{ergs} \mathrm{s}^{-1}, \approx 8 \pm 2 \%$ of GCs contain an LMXB within $1^{\prime \prime}$ after correcting for random matches. Among all detected sources $\left(L_{X}>0.6 \times 10^{37} \mathrm{ergs} \mathrm{s}^{-1}\right)$, this rises to $\approx 10 \pm 2 \%$. Since active LMXBs can have $L_{X} \gtrsim 10^{36} \mathrm{ergs} \mathrm{s}^{-1}$, it is likely that the percentage of GCs with an active LMXB is even higher.

Over the history of X-ray astronomy, 12/150 MW-GCs have had an active LMXB (Liu, van Paradijs, \& van den Heuvel 2001; Harris 1996). This corresponds to $\approx 8_{-2}^{+3} \%$ of GCs having contained an active LMXB. Since some LMXBs in Galactic GCs are transient, this is an upper limit to the instantaneous percentage of GCs containing active LMXBs.

It is likely that the percentage of GCs with an active LMXB in NGC 4697 will be higher than that of the MW. Given that NGC 4697 has a higher fraction of red GCs to blue GCs than the MW (Jordán et al. 2005) and that red GCs appear to be more likely to contain an LMXB (e.g., Sarazin et al. 2003), this result is not unexpected.

\section{Variability Results}

Milky Way LMXBs exhibit a wide range of variability, including transient outbursts, Type I bursts, and milder fluctuations. Such behavior is expected from LMXBs in E/S0s.

In Sivakoff, Sarazin, \& Jordán (2005), we discussed the detection of flaring sources in NGC 4697 using a new technique. Two sources show $\sim 1000$ s flares with $L_{\text {bol }}>4 \times$ $10^{38} \mathrm{ergs} \mathrm{s}^{-1}$. Although the timescale of the flares is similar to superbursts, the luminosity is higher than expected for a neutron star (NS). Furthermore, the recurrence timescale of flares ( $\lesssim$ days) is much shorter than the approximately year-long timescale of Galactic superbursts. An even more perplexing source with recurrent $\sim 100$ s flares was also found (e.g., Fig. 2 left). The flaring in this source is most like that of LMC X-4. However, the flaring mechanism in LMC X-4 requires a very strong magnetic field (Moon, Eikenberry, \& Wasserman 2003). Such an interpretation would require that accretion is the source of magnetic field decay and that the NGC 4697 source recently began accretion. The source is also similar to V4641 Sgr, a HMXB with a radio jet inclined only $\sim 15^{\circ}$ to the lineof-sight. The flaring in the source in NGC 4697 could indicate that it is a micro-blazar. 

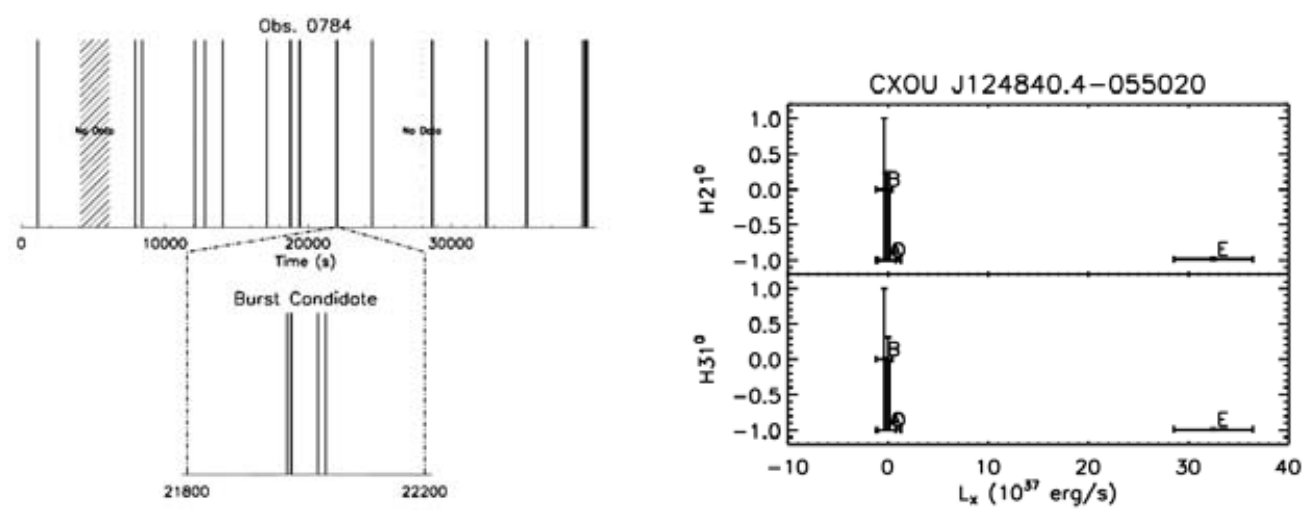

Figure 2. (Left) Impulse diagram indicating the time of arrival of photons in flaring source CXOU J124839.0-054750. (Right) Luminosity versus hardness ratio of a supersoft transient.

A variety of Galactic LMXBs exhibit long term variability (LTV), including Z/Atoll NS-LMXBs, soft X-ray transients, long-term transients like GRS1915+105, and black hole $(\mathrm{BH})$ state changes. We detect LTV for 26/124 sources in NGC 4697. Of these sources, $11 / 26$ are transient candidates. Assuming these transients are long-term transients, we calculate a mean outburst time of 105 yrs. One candidate is clearly a supersoft transient (Fig. 2 right). It goes from being undetected to $L_{\mathrm{bol}} \approx 8 \times 10^{38} \mathrm{ergs} \mathrm{s}^{-1}$ with a disk blackbody temperature of $\approx 0.14 \mathrm{keV}$. From its temperature, such a source could be interpreted as an intermediate-mass $\mathrm{BH}$; however, this would require the source to be accreting well below its Eddington limit. We have also found evidence for a source showing a luminosity/spectral state transition. The source goes from being soft and faint to hard and bright, opposite of the conventional relationship in $\mathrm{BH}$ state transitions. It is possible that this discrepancy results from the softer bands used to calculate the hardness with Chandra; the Chandra soft state might result from the same power law spectrum as the RXTE hard state (Juett et al. 2005, in preparation).

\section{Acknowledgements}

Support for this work was provided by NASA through HST Award GO-10003.01-A and Chandra Awards GO5-6086X, GO4-5093X, AR3-4005X, GO3-4099X, and AR4-5008X.

\section{References}

Angelini, L., Loewenstein, M., \& Mushotzky, R. F. 2001, ApJ 557, L35

Côté, P., et al. 2004, ApJS, 153, 223

Giacconi, R., Gursky, H., Paolini, F. R., \& Rossi, B. B. 1962, Phys Rev Lett 9439

Harris, W. E. 1996, AJ, 112, 1487

Jordán, A., et al. 2004, ApJS, 154, 509

Liu, Q. Z., van Paradijs, J., \& van den Heuvel, E. P. J. 2001, A\&A, 368, 1021

Kim, D.-W., et al. 2004, ApJ, 600, 59

Kim, D.-W. \& Fabbiano, G. 2004, ApJ, 611, 846

Kim, D.-W., Fabbiano, G., \& Trinchieri, G. 1992, ApJ 393, 134

Moon, D.-S., Eikenberry, \& Wasserman, I. M. 2003, Apj 586, 1280

Sarazin, C. L., Irwin, J. A., \& Bregman, J. N. 2000, ApJ 544, L101

Sarazin, C. L., Irwin, J. A., \& Bregman, J. N. 2001, ApJ 556, 533

Sarazin, C. L., Kundu, A., Irwin, J. A., Sivakoff, G. R., Blanton, E. L., \& Randall, S. W. 2003, ApJ 595, 743

Sivakoff, G. R., Sarazin, C. L., \& Jordán, A. 2005, ApJ 624, L17

Sivakoff, G. R., Sarazin, C. L., \& Irwin, J. A. 2003, ApJ 599, 218 


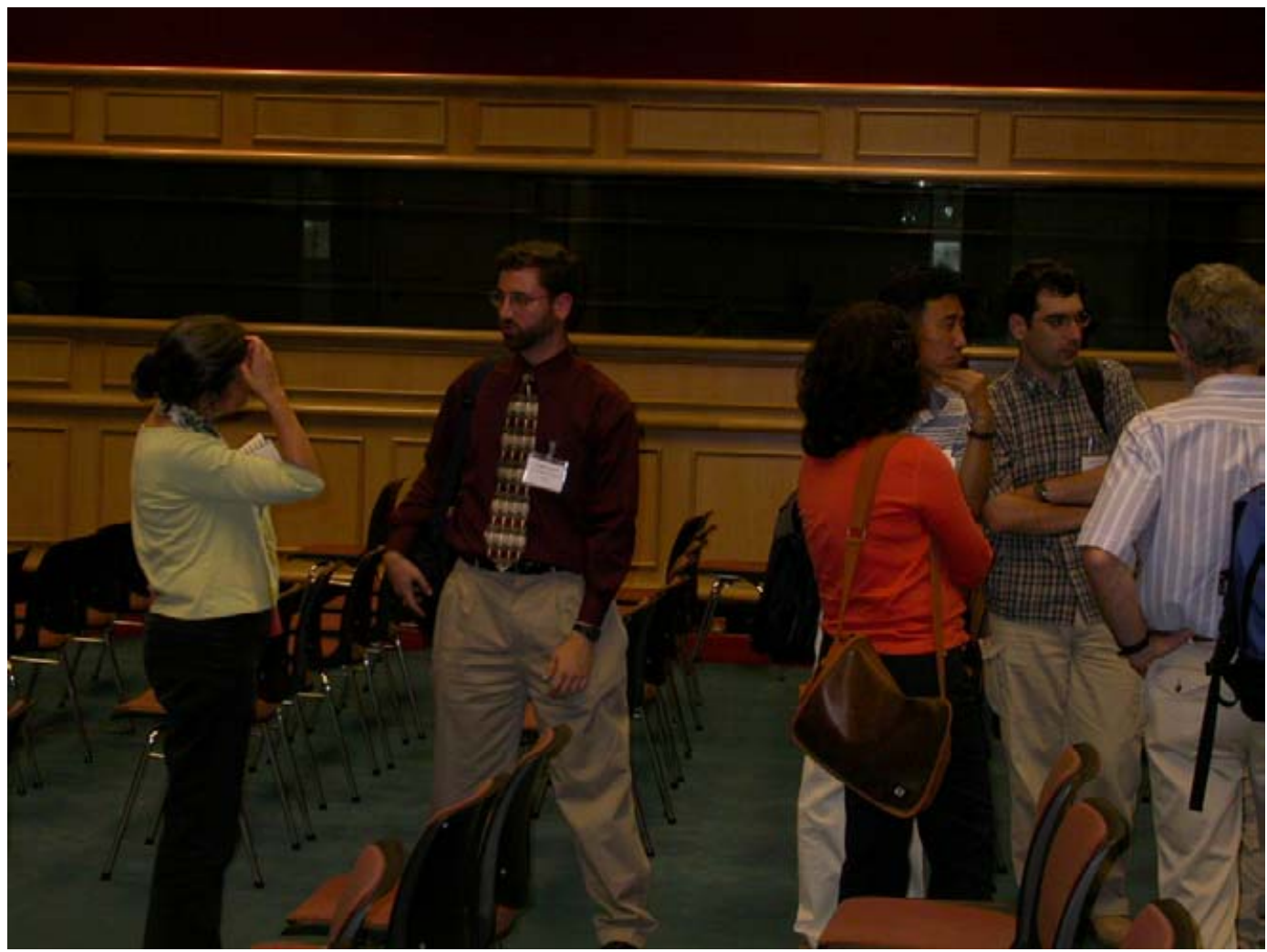

After the 2nd Session. Gregory Sivakoff (in the middle) has just presented his contribution.

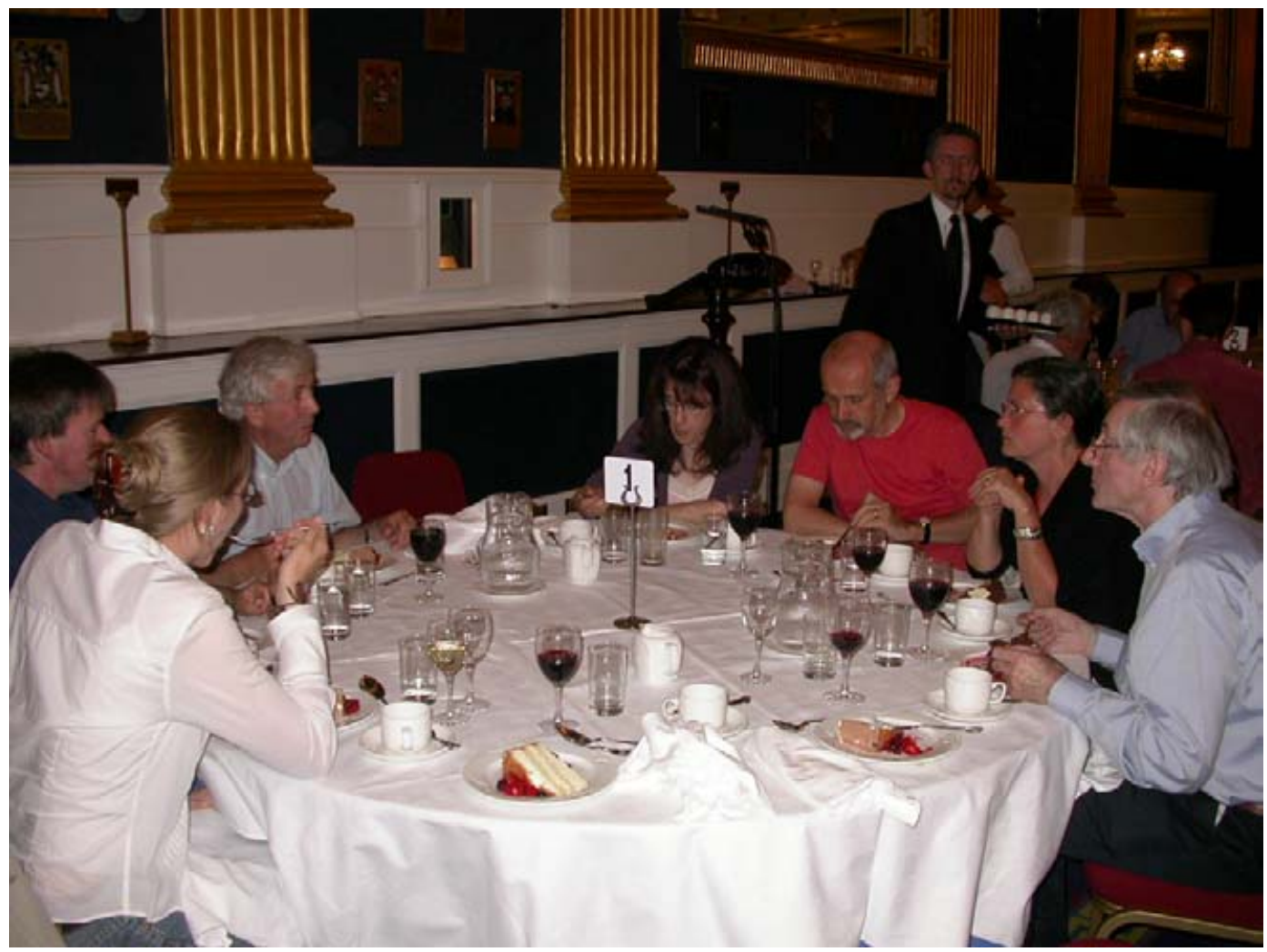

Conference Dinner - at the dessert. Notice the forest of glasses. From left to right: Kevin Nolan, Ines Brott, Brian McBreen, Laura Norci, Martin Elvis, Pepi Fabbiano, George Miley. 